\title{
Overexpression and gene amplification of PD-L1 in cancer cells and PD-L1+ immune cells in Epstein-Barr virus-associated gastric cancer: the prognostic implications
}

Ruri Saito ${ }^{1}$, Hiroyuki Abe ${ }^{2}$, Akiko Kunita ${ }^{1}$, Hiroharu Yamashita ${ }^{3}$, Yasuyuki Seto ${ }^{3}$ and Masashi Fukayama ${ }^{1,2}$

${ }^{1}$ Department of Pathology, Graduate School of Medicine, The University of Tokyo, Tokyo, Japan; ${ }^{2}$ Department of Pathology, The University of Tokyo Hospital, Tokyo, Japan and ${ }^{3}$ Department of Gastrointestinal Surgery, Graduate School of Medicine, The University of Tokyo, Tokyo, Japan

Cancer cells use PD-L1 to evade antitumor immunity through interaction with programmed cell death protein 1 (PD-1) on T cells. Recent whole-genome sequence studies revealed frequent gene amplification of $P D-L 1$ in Epstein-Barr virus-associated gastric cancer (EBVaGC). To investigate the significance of PD-L1 in cancer cells and their microenvironment in EBVaGC, we studied PD-L1 expression by analysis of the public database and immunohistochemistry with fluorescent in situ hybridization (FISH) of the PD-L1 gene. Analysis of the database from The Cancer Genome Atlas also disclosed high expression of PD-L1 in EBVaGC compared with other molecular subtypes of GC. Expression of PD-L1 was frequently detected in cancer cells of EBVaGC (33/96; 34\%), with infiltration of PD-L1+ immune cells in its stroma (43/96; 45\%). Both expression of PD-L1 in cancer cells and PD-L1+ immune cell infiltration in EBVaGC were significantly correlated with diffuse histology according to Lauren's classification and tumor invasion (pT1b or more). As a prognostic indicator, PD-L1 expression in cancer cells correlated with poor outcomes in both overall survival and disease-specific survival $(P=0.0498,0.007)$. PDL1-positive cancers had dense infiltration of PD-L1+ immune cells as well as CD8 ${ }^{+}$and PD-1+ cells in EBVaGC. FISH analysis of representative samples of the tumor demonstrated gene amplification of $P D-L 1$ in $11 \%$ of cases. PD-L1-amplified cells corresponded to PD-L1-positive cells showing high-intensity immunohistochemical staining among cancer cells showing weak or moderate intensities. Taken together, PD-L1 expression in cancer cells and their microenvironment may contribute to the progression of EBVaGC, and gene amplification occurs as clonal evolution during progression. This specific subtype of GC infected with EBV is potentially a good candidate for immunotherapy targeting of the PD-L1/PD-1 axis.

Modern Pathology (2017) 30, 427-439; doi:10.1038/modpathol.2016.202; published online 9 December 2016

Gastric cancer (GC) is one of the major causes of cancer-related death in the world. There are various histological types of GC, which correlate with different biological characteristics. ${ }^{1}$ Several groups recently reported the results of comprehensive genome analyses, ${ }^{2,3}$ and the Cancer Genome Atlas Research Network (TCGA) proposed four molecular subtypes of GC in 2014: Epstein-Barr virus (EBV)positive, microsatellite instability (MSI), genomically

Correspondence: Professor M Fukayama, MD, PhD, Department of Pathology, Graduate School of Medicine, The University of Tokyo, Hongo 7-3-1, Tokyo 113-0033, Japan.

E-mail: mfukayama-tky@umin.org

Received 23 June 2016; revised 7 September 2016; accepted 18 October 2016; published online 9 December 2016 stable (GS), and chromosomal instability (CIN) subgroups. $^{2}$ The MSI subgroup was characterized by hypermutation with promoter methylation and suppression of $M L H 1$, one of the mismatch repair genes. Most GS subgroup cancers correspond to histologically diffuse phenotype, whereas CIN subgroup cancers frequently show intestinal-type histology.

EBV-associated GC (EBVaGC), which corresponds to the EBV-positive group in the TCGA study, is a distinct subtype of GC. EBVaGC consists of monoclonal tumor cells infected with EBV, and it accounts for $\sim 5-10 \%$ of GCs. The histology of EBVaGC is poorly to moderately differentiated adenocarcinoma, often accompanied by dense lymphocytic infiltration. ${ }^{4-7}$ Comprehensive molecular analyses 
have revealed characteristic epigenetic abnormalities associated with EBVaGC, that is, genome-wide methylation of $\mathrm{CpG}$ islands in the promoter region of various tumor suppressor genes. ${ }^{8,9}$ In addition, some genomic abnormalities, such as mutation of the PIK3CA, ARID1A, and BCOR genes, are also frequent characteristics of EBVaGC. Amplification of 9p24.1, which contains the PD-L1, PD-L2, and JAK2 genes, occurs in $\sim 15 \%$ of EBVaGC cases. ${ }^{2}$

PD-L1 is a ligand of programmed cell death protein 1 (PD-1), which is expressed on T cells. PD-L1, which is expressed on tumor cells or stromal immune cells, inhibits activation of cytotoxic $\mathrm{T}$ cells through an interaction with PD-1, and helps cancer cells to evade antitumor immunity. ${ }^{10-12}$ As expression of PD-L1 is observed in many malignant tumors and is associated with poor prognosis, PD-L1 has been studied extensively as a therapeutic target, especially in melanoma and non-small-cell lung cancer. ${ }^{13-17}$ Several studies have demonstrated that PD-L1, expressed on cancer cells or tumorinfiltrating immune cells, is a prognostic factor in GC, but the significance of PD-L1 in EBVaGC has not yet been clarified. ${ }^{18-22}$

To determine the clinicopathological significance of PD-L1 in EBVaGC compared with other subtypes of GC, the public database of TCGA was analyzed for PD-L1 mRNA expression. Then, we performed immunohistochemistry of PD-L1 using a large sample of EBVaGC cases. In addition, we performed fluorescence in situ hybridization (FISH) to detect gene amplification of $P D-L 1$ in EBVaGC.

\section{Materials and methods}

\section{Analysis of PD-L1 mRNA Expression in the Public Database of TCGA}

PD-L1 mRNA expression data was extracted from TCGA with cBioPortal for Cancer Genomics website, which provides analysis of large-scale cancer genomics data sets. ${ }^{2,23,24}$ The expression data was plotted in a box-plot chart and compared between four molecular subtypes of GC: tumors with chromosomal instability (CIN), EBV-positive tumors, GS tumors, and MSI tumors.

\section{Tissue Samples}

Formalin-fixed, paraffin-embedded GC tumor tissue specimens derived from 232 cases of GC from the archive at the Department of Pathology, The University of Tokyo Hospital (Tokyo, Japan). These cases included 96 EBVaGC and 136 EBV-negative GC. The EBVaGC cases, which were positive for EBV-encoded small RNAs (EBERs)-in situ hybridization, were consecutive cases resected between 1990 and 2013. EBV-negative GC cases included three groups. One group, MLH1-negative by immunohistochemistry, comprised 36 consecutive cases resected between 2003 and 2007 and corresponded to tumors with MSI. The other two groups, EBERnegative and MLH1-positive, comprised 100 consecutive cases resected in 2005. These cases were classified by the histological type (diffuse and intestinal) according to Lauren's histological classification. ${ }^{25}$ Clinicopathological information was obtained from medical records. Tissue microarrays (TMAs) were designed from the paraffin tissue blocks of GC by punching out two 2-mm-diameter cores from each block.

This study was approved by the Ethics Committee of the institution (Graduate School of Medicine, The University of Tokyo, Tokyo, Japan).

\section{Classification of GCs Based on EBER-In Situ Hybridization and MLH1 Immunohistochemistry}

Based on the molecular classification proposed by the TCGA research group, four subtypes of GC were defined by EBER-in situ hybridization, MLH1 immunohistochemistry, and histological type (diffuse and intestinal). The TMAs, described above, were used for this purpose. To classify the GCs, EBER-in situ hybridization was performed with a fluorescein isothiocyanate (FITC)-labeled peptide nucleic acid probe (Y5200; Dako, Glostrup, Denmark), followed by immunohistochemistry with an anti-FITC antibody (V0403, dilution 1:200; Dako). MLH1 immunohistochemistry was applied to TMAs using a mouse monoclonal anti-MLH1 (clone ES05, dilution 1:50; Leica, Wetzlar, Germany). In situ hybridization and immunohistochemistry were performed with a Ventana Benchmark automated immunostainer (Ventana Medical Systems, Tuscon, AZ, USA) according to the manufacturer's protocols.

\section{Immunohistochemistry of PD-L1, CD8, and PD-1}

Immunohistochemistry of PD-L1, CD8, and PD-1 was performed on whole sections from each tumor. One representative block was selected from each tumor and sliced into $3-\mu \mathrm{m}$-thick sections, which were then immunostained with the Ventana Benchmark automated immunostainer. The primary antibodies used were rabbit monoclonal anti-PD-L1 antibodies (clone E1L3N, dilution 1:200; Cell Signaling Technology, Danvers, MA, USA), mouse monoclonal anti-CD8 antibodies (clone 4B11, dilution 1:40; Leica), and mouse monoclonal anti-PD-1 antibodies (clone NAT105, dilution 1:100; Abcam, Cambridge, UK).

Immunohistochemical results were evaluated independently by two pathologists (RS and HA), and slides were reinvestigated by both observers using a multiheaded microscope when there was a difference between the two evaluations. Immunohistochemistry of PD-L1 was assessed by cell membrane staining, and cancer cells that stained only in the cytoplasm were regarded as negative. Cancer cell staining was classified into four proportion scores 
( $P$-scores) according to the expression status of $\mathrm{PD}$ L1 as follows: score 0 , no stained cancer cells in the section; score $1, \leq 5 \%$ of tumor cells stained in the cell membrane; score $2,>5 \%$; and score $3,>50 \%$. For the correlation analysis, $P$-scores of 0 and 1 were regarded as negative, whereas $P$-scores of 2 and 3 were regarded as positive. The intensity of immunohistochemistry was scored as an intensity score (Iscore) 0 (no staining), 1 (weak to intermediate intensity), and 2 (high intensity). The expression of PD-L1 in stromal immune cells in the tumor tissues was also evaluated and simply classified into negative or positive groups depending on the proportion of stained cells (cutoff value: $1 \%$ ).

Immunohistochemistry of CD8 was performed for 77 cases of EBVaGC that contained invasive areas in the submucosa or deeper. Evaluation of the infiltration of CD8-positive cytotoxic T cells, along with digital image analysis, was recently published by the authors, ${ }^{26}$ and these data were used in the present study to examine the correlation between PD-L1 expression and CD8-positive cells. In brief, tissue sections were analyzed by immunohistochemistry, then digitized using a Nanozoomer 2.0-HT virtual slide scanner (Hamamatsu Photonics, Hamamatsu, Japan), and the digital images were analyzed using the Tissue Studio software (Definiens, Munich, Germany). The area encompassed by the invasive tumor was circled by an experienced pathologist (HA). The percentage of positive staining within this circled area was measured automatically using the Tissue Studio software.

As for the evaluation of PD-1-positive cells, digital image analysis was not applied, as it was sometimes difficult to discriminate tumor cells from inflammatory cells using this software. Instead, 77 cases were divided into three groups semiquantitatively depending on the intensity of PD-1-positive cell infiltration: 0 , mild infiltration of PD-1-positive cells; $1+$, moderate infiltration; and $2+$, severe infiltration. 0 was regarded as negative, whereas $1+$ and $2+$ were regarded as positive.

\section{Fluorescence In Situ Hybridization}

TMAs containing 44 consecutive cases of EBVaGC were sliced into $4-\mu \mathrm{m}$-thick sections for FISH, which was performed according to the protocol provided by the GSP-Lab (Kawasaki, Japan). Briefly, after deparaffinization the slides were boiled for $30 \mathrm{~min}$ in pretreatment solution at $100{ }^{\circ} \mathrm{C}$. The slides were washed and dipped for $15 \mathrm{~min}$ in enzyme solution at $37^{\circ} \mathrm{C}$. After washing and drying slides, hybridization with probes (CD274/CEN9q Dual-Color FISH Probe; GSP-Lab) was carried out in a light-shielded wet box at $37^{\circ} \mathrm{C}$ overnight. After staining nuclei with $4^{\prime}, 6$ diamidino-2-phenylindole, signals were observed with a fluorescence microscope (DM6000B; Leica). Only nuclei with clear CEN9q signals were included, and overlapping nuclei were excluded from the count to avoid false-positive results. At least 10 cells were counted, and the CD274/CEN9q ratio was calculated in each tumor. If the CD274/CEN9q ratio was $\geq 2$, the tumor was considered as positive for gene amplification of PD-L1. Adjacent slides of the same TMAs were subject to immunohistochemical staining of PD-L1, and the results of immunohistochemistry and FISH were compared with each other.

FISH was also carried out with the same method using whole sections of the tumors, which contained clusters of PD-L1-positive cells with an I-score of 2 for the immunostained slides. In addition, FISH was performed in EBV-negative GC with positive expression of PD-L1.

\section{Statistical Analysis}

The relationships between expression of PD-L1 and clinicopathological factors (patients' age, sex, tumor location, tumor size, histological type, tumor invasion depth, venous or lymphatic invasion, and lymph node metastasis) or infiltration of PD-1positive cells were examined by a $\chi^{2}$ test. Continuous variables such as patients' age, tumor size, and the extent of CD8-positive cell infiltration were also analyzed by Student's $t$-test. Overall survival (OS) was measured from the time of resection to the time of death from any cause or the time of the last followup. Disease-specific survival (DSS) was calculated from the time of resection to the time of death from GC or the time of last follow-up. OS and DSS were plotted by Kaplan-Meier curves, and differences between PD-L1-positive and -negative cases were evaluated by the log-rank test. Multivariate analyses of survival were performed with the Cox proportional hazards model. A backward elimination was used with a threshold of $P=0.10$ to select variables for the final model. The comparison of PD-L1 expression in EBVaGC and EBV-negative GC cases was performed by a $\chi^{2}$ test. These statistical analyses were conducted using the JMP Pro 11 software package (SAS Institute, Cary, NC, USA). $P$-values $<0.05$ were regarded as statistically significant.

\section{Results}

\section{Analysis of PD-L1 mRNA Expression Data from the Public Database of TCGA}

We analyzed the public database of TCGA to determine whether EBVaGC expresses higher level of PD-L1 mRNA than other GC. The data was summarized in a box-plot chart and shown in Figure 1. EBVaGC $(n=24)$ had higher level of PDL1 mRNA compared with other three molecular subtypes (CIN: $n=128$; GS: $n=54$; MSI: $n=59$ ) (EBV vs CIN, $P<0.001$; EBV vs GS, $P<0.001$; EBV vs MSI, $P<0.001)$. 


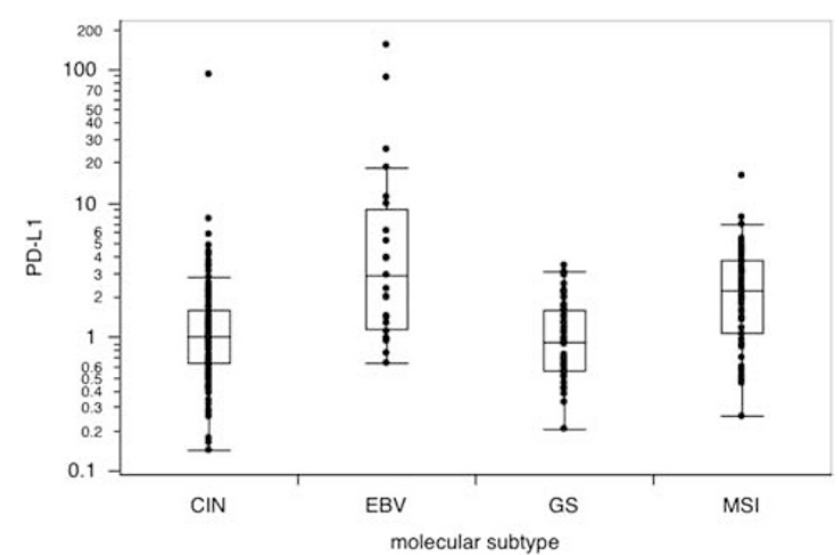

Figure 1 Programmed death-ligand 1 (PD-L1) mRNA expression data from The Cancer Genome Atlas Network (TCGA). Data of PDL1 mRNA expression in gastric cancer was extracted from the public database of TCGA and compared among the four molecular subtypes (chromosomal instability (CIN), EBV-positive (EBV), genomically stable (GS) and microsatellite instability (MSI)). EBV group showed significantly higher expression level than other three groups.

\section{Immunohistochemistry}

Immunohistochemistry was first used for the evaluation of PD-L1 expression, as well as $\mathrm{CD}^{+}$and $\mathrm{PD}-1^{+}$ immune cell infiltration. PD-L1 immunostaining was noted in the cell membrane of cancer cells and was generally weak in stromal immune cells. Representative images of PD-L1 immunohistochemical staining are presented in Figure 2. Regarding the evaluation of staining, the concordance rate between two pathologists was nearly $95 \%$.

\section{PD-L1 Expression in Cancer Cells of EBVaGC and EBV-Negative GC}

The frequency of PD-L1 expression in cancer cells was $34.4 \%$ (33/96) in EBVaGC, comprising 26 cases with a P-score of 2 and seven cases with a $P$-score of 3 (Table 1). The intensity of staining among positive immunoreactive EBVaGC cells was generally homogenous and of weak to moderate strength (I-score 1), but some positive cells of high intensity (I-score 2) formed scattered clusters of several cells in seven cases ( $P$-score 2 and 3 ; one and six cases) or sheets of many cells in four cases ( $P$-score 2 and 3 ; three and one cases). By contrast, $4.4 \%$ of EBV-negative GC cases (6/136) showed a $P$-score of 2 , and none of the positive cells showed an $I$-score of 2 . The frequency of PD-L1 positivity in cancer cells was $5.6 \%$ for the MLH1-negative subtype (2/36), 6.0\% for the diffuse subtype $(3 / 50)$, and $2.0 \%$ for the intestinal subtype $(1 / 50)$.

\section{PD-L1 Expression in Immune Cells of EBVaGC and EBV-Negative GC}

The frequency of PD-L1 expression in immune cells in the cancer stroma was generally weak and comprised a small number of cells. However, when the cutoff value was set at $1 \%$, the frequency was higher in EBVaGC $(44.8 \%, 43 / 96)$ compared with that in EBV-negative GC $(27.2 \%, 37 / 136)$ $(P=0.006) \quad$ (Table 1). PD-L1 ${ }^{+}$immune cells were also frequently detected in the MLH1-negative subtype $(44 \%, 16 / 36)$, followed by the intestinal $(24 \%, 12 / 50)$ and diffuse subtypes $(18 \%, 9 / 50)$ (Table 1).

\section{Clinicopathological Significance of PD-L1 Expression in EBVaGC}

The expression of PD-L1 in cancer cells in EBVaGC was significantly correlated with diffuse histology according to Lauren's classification $(P=0.002)$ and tumor invasion into the submucosa or deeper (pT1b or more) $(P=0.014)$, whereas expression of PD-L1 in immune cells in EBVaGC was significantly correlated with diffuse histology $(P=0.006)$, tumor invasion $(P<0.001)$, and the presence of venous and lymphatic invasion $(P=0.005$ and $P=0.014$, respectively) (Table 2).

As for the correlation between immune cell infiltration and PD-L1 expression in cancer cells of EBVaGC, infiltration of both $\mathrm{CD}^{+}$and $\mathrm{PD}-1^{+}$cells were significantly more frequent in PD-L1-positive cases than in PD-L1-negative cases $(P<0.001$ and $P=0.020$, respectively) (Figure 3, and Table 3). PD-L1 expression in stromal cells was also significantly associated with PD-L1 expression in cancer cells (Table 3). Infiltration of PD-1 ${ }^{+}$cells was relatively high in GCs located in the middle or lower regions of the stomach, but otherwise there was no correlation with other clinicopathological factors (Supplementary Table 1). As for the infiltration of $\mathrm{CD}^{+}$cells, as reported in the previous study, ${ }^{26}$ low infiltration occurred in the upper part of the stomach and correlated with an advanced tumor stage.

\section{Clinicopathological Significance of PD-L1 Expression in EBV-Negative GC}

The expression of PD-L1 in immune cells was relatively frequent compared with that in cancer cells, and therefore its correlation with clinicopathological factors was evaluated according to the subtype of EBV-negative GC (Supplementary Table 2). The frequency of PD-L1 expression in the MLH1negative subtype was comparable with that in EBVaGC, but there was no significant correlation between PD-L1 ${ }^{+}$immune cell infiltration and clinicopathological factors. Lymph node metastases, however, were more frequent in the cases infiltrated by PD-L1 $1^{+}$immune cells in both the intestinal and diffuse subtypes of GC (Supplementary Table 2). 

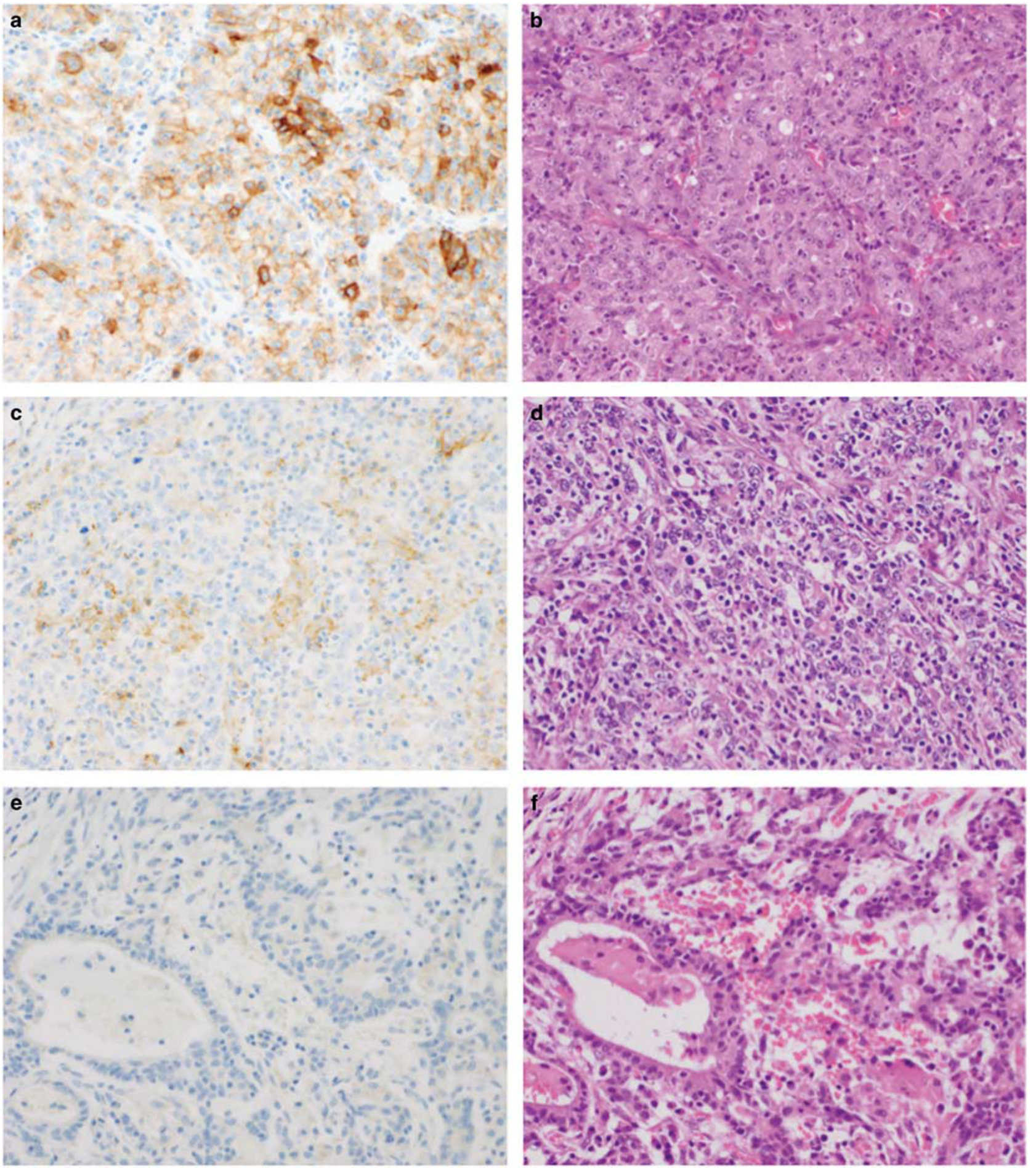

Figure 2 Programmed death-ligand 1 (PD-L1) immunohistochemical staining of gastric cancer tissue. Immunohistochemical staining of PD-L1 (a, c, and $\mathbf{e})$ and hematoxylin-eosin staining (b, d, and $\mathbf{f}$ ) of the same region of gastric cancer tissue. Expression of PD-L1 was evaluated in the cell membrane. P-score 3, moderate to strong staining ( $I$-score 1 or 2 ) was observed diffusely (a). $P$-score 2 , moderate to strong staining ( $I$-score 1 or 2 ) was observed in $>5 \%$ of cells (c). P-score 0 , no stained cells detected (e). Some immune cells also express PD-L1 weakly in (a) and (c), whereas expression of PD-L1 in immune cells is not observed in (e).

\section{PD-L1 Expression as a Prognostic Indicator for EBVaGC}

Cases that displayed PD-L1-positive cancer cells were associated with poorer outcomes for both OS
$(P=0.0498)$ and DSS $(P=0.007)$ (Figures $4 \mathrm{a}$ and $\mathrm{b})$. In multivariate analysis with the Cox proportional hazards model, only lymph node metastasis showed a significant correlation with OS. The expression of PD-L1 showed a tendency towards a poorer 
Table 1 Comparison of PD-L1 expression in EBV-associated and EBV-negative gastric cancer

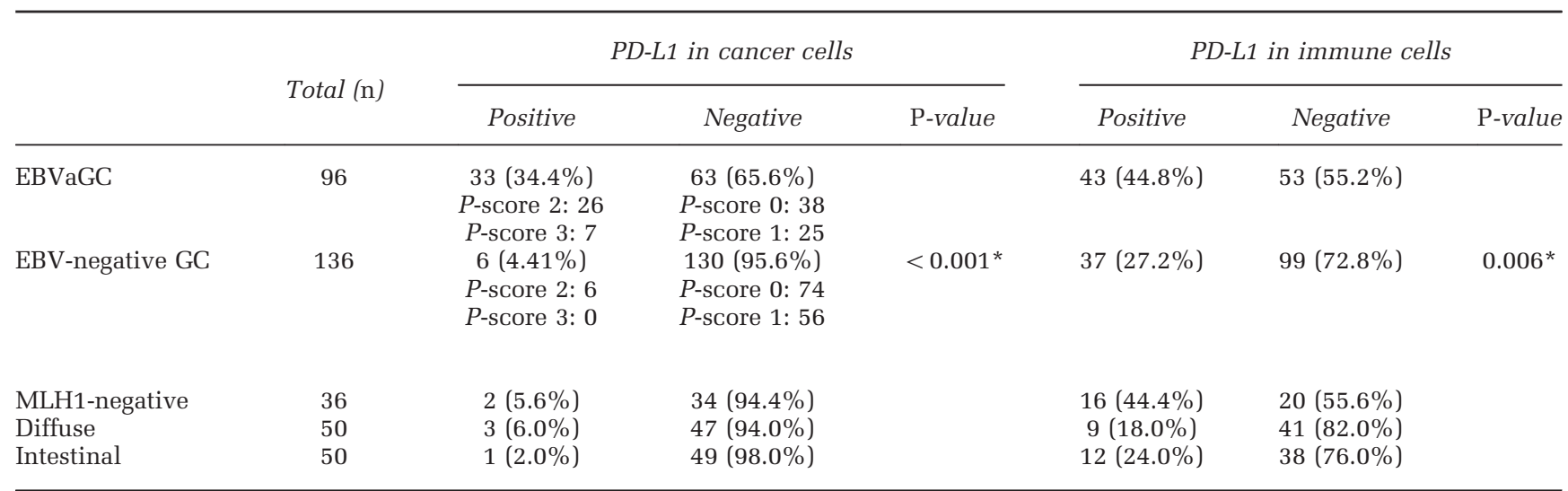

Abbreviations: EBV, Epstein-Barr virus; EBVaGC, EBV-associated gastric cancer; GC: gastric cancer; PD-L1, programmed death-ligand 1; P-score, proportion score.

${ }^{*} P<0.05$.

Table 2 PD-L1 expression and clinicopathological characteristics of EBV-associated gastric cancer

\begin{tabular}{|c|c|c|c|c|c|c|}
\hline \multirow{2}{*}{ Characteristic } & \multicolumn{2}{|c|}{$P D-L 1$ in cancer cells } & \multirow[b]{2}{*}{ P-value } & \multicolumn{2}{|c|}{$P D-L 1$ in immune cells } & \multirow[b]{2}{*}{$\mathrm{P}$-value } \\
\hline & Positive & Negative & & Positive & Negative & \\
\hline All cases & $33(34.4 \%)$ & $63(65.6 \%)$ & & $43(44.8 \%)$ & $53(55.2 \%)$ & \\
\hline \multicolumn{7}{|l|}{ Patients' age } \\
\hline Median (range) & $65(40-90)$ & $64(40-87)$ & 0.209 & $69(40-90)$ & $63(40-87)$ & 0.135 \\
\hline$>65 / \leq 65$ & $15 / 18$ & $28 / 35$ & 0.925 & $23 / 20$ & $20 / 33$ & 0.123 \\
\hline \multicolumn{7}{|l|}{ Sex } \\
\hline Male/female & $26 / 7$ & $50 / 13$ & 0.947 & $32 / 11$ & $44 / 9$ & 0.302 \\
\hline \multicolumn{7}{|l|}{ Tumor location } \\
\hline $\mathrm{U} / \mathrm{M} / \mathrm{L}$ & $17 / 13 / 3$ & $34 / 22 / 7$ & 0.892 & $19 / 19 / 5$ & $32 / 16 / 5$ & 0.278 \\
\hline \multicolumn{7}{|l|}{ Tumor size } \\
\hline Median (range) & $45(8-180)$ & $40(13-200)$ & 0.183 & $50(8-180)$ & $40(10-200)$ & 0.077 \\
\hline$>40 \mathrm{~mm} / \leq 40 \mathrm{~mm}$ & $18 / 15$ & $30 / 33$ & 0.519 & $26 / 17$ & $22 / 31$ & 0.065 \\
\hline \multicolumn{7}{|l|}{ Histological type } \\
\hline Intestinal/diffuse & $8 / 25$ & $36 / 27$ & $0.002^{*}$ & $13 / 30$ & $31 / 22$ & $0.006^{*}$ \\
\hline \multicolumn{7}{|l|}{ Tumor invasion depth } \\
\hline pT1a/pT1b+pT2+pT3+pT4 & $1 / 32$ & $14 / 49 /$ & $0.014^{*}$ & $0 / 43$ & $15 / 38$ & $<0.001^{*}$ \\
\hline \multicolumn{7}{|l|}{ Venous invasion } \\
\hline Absent/present & $13 / 20$ & $38 / 25$ & 0.051 & $16 / 27$ & $35 / 18$ & $0.005^{*}$ \\
\hline \multicolumn{7}{|l|}{ Lymphatic invasion } \\
\hline Absent/present & $19 / 14$ & $45 / 18$ & 0.171 & $23 / 20$ & $41 / 12$ & $0.014^{*}$ \\
\hline \multicolumn{7}{|l|}{ Lymph node metastasis } \\
\hline Absent/present & $21 / 12$ & $46 / 17$ & 0.342 & $26 / 17$ & $41 / 12$ & 0.073 \\
\hline
\end{tabular}

Abbreviations: EBV, Epstein-Barr virus; PD-L1, programmed death-ligand 1; L, lower; M, middle; U, upper.

${ }^{*} P<0.05$.

prognosis, although this was not statistically significant (Table 4). As the number of events was too few, we could not perform multivariate analysis of DSS. Cases displaying PD-L1 expression in immune cells also showed a tendency towards poorer outcomes for both OS and DSS, although this was not statistically significant $(P=0.1192,0.0521)$ (Figures 4c and d). 

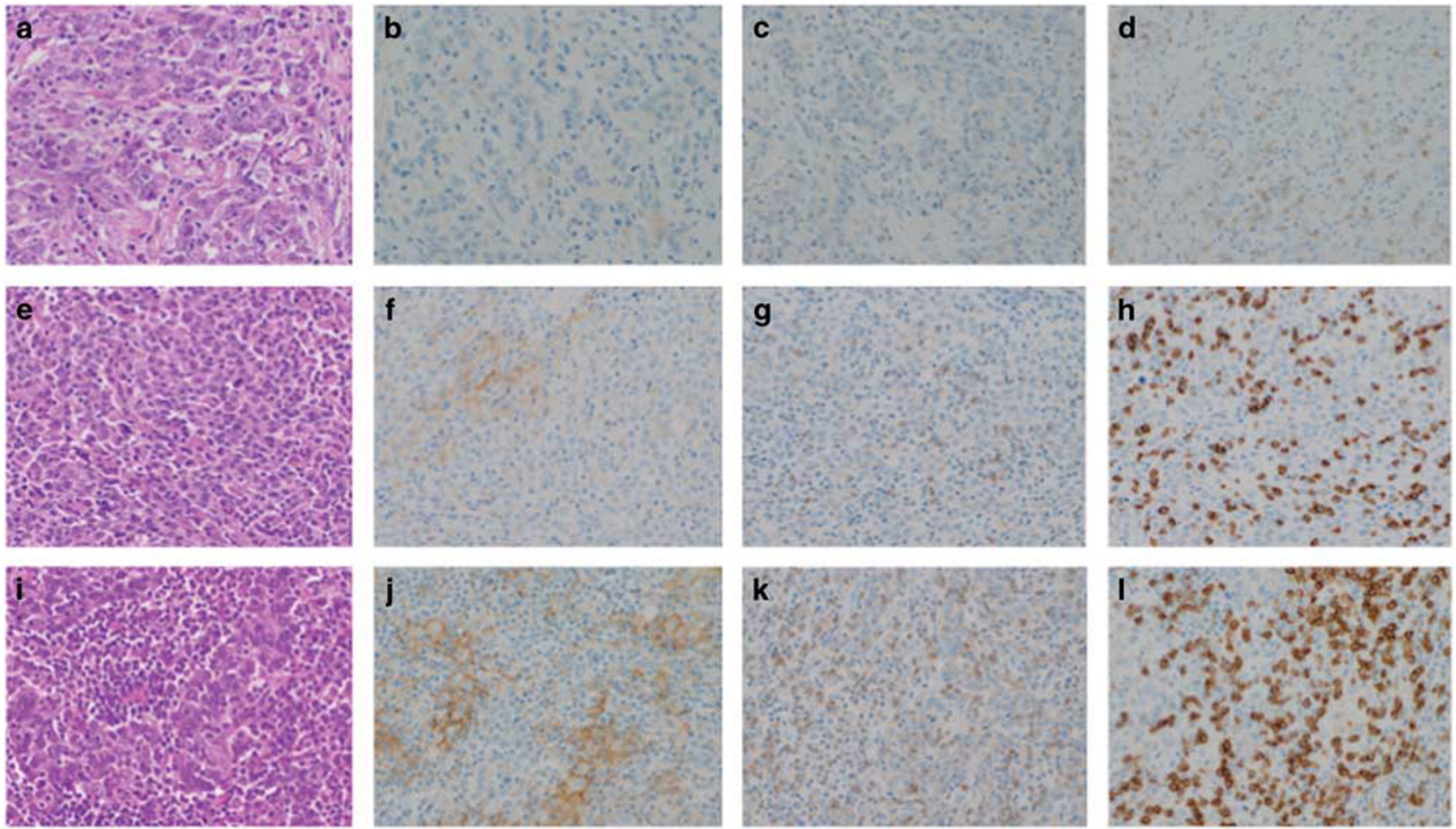

Figure 3 Expression of programmed death-ligand 1 (PD-L1) in cancer cells and infiltration of PD-1 $1^{+}$or CD8 ${ }^{+}$inflammatory cells in Epstein-Barr virus-associated gastric cancer (EBVaGC) tissue. EBVaGC tissue showed positive expression of PD-L1 accompanied by dense infiltration of PD- $1^{+}$or $\mathrm{CD}^{+}$inflammatory cells within the tumor. (a-d) A case that showed no expression of PD-L1 in cancer cells (P-score $0, I$-score 0). (e-h) A case with a $P$-score of 2 and an $I$-score of 1 for expression of PD-L1 in cancer cells. (i-l) A case with a $P$-score of 3 and an $I$-score of 2 for expression of PD-L1 in cancer cells. (a, e, and i) Hematoxylin and eosin staining; (b, f, and j): PD-L1 immunostaining; (c, g, and k): PD-1 immunostaining; (d, h, and l): CD8 immunostaining.

Table 3 Relationships between PD-L1 expression in cancer cells and various stromal immune cells

PD-L1 in cancer cells

\begin{tabular}{|c|c|c|c|}
\hline & & \multirow[b]{2}{*}{ P-value } \\
\hline & Positive (P-score 2, 3) & Negative (P-score 0,1$)$ & \\
\hline$P D-L 1$ in stromal cells $(\mathrm{n}=96)$ & & & $<0.001^{*}$ \\
\hline Positive & 31 & 12 & \\
\hline Negative & 2 & 51 & \\
\hline$P D-1(\mathrm{n}=77)$ & & & $0.020^{*}$ \\
\hline Positive $(1+: 31 ; 2+: 5)$ & 20 & 16 & \\
\hline Negative (0: 41) & 12 & 29 & \\
\hline CD8-positive cells (\%) ${ }^{\mathrm{a}}$ & $27.77 \pm 9.88$ & $12.34 \pm 8.78$ & $<0.001^{*}$ \\
\hline
\end{tabular}

Abbreviations: PD-L1, programmed death-ligand 1; P-score, proportion score.

${ }^{*} P<0.05$.

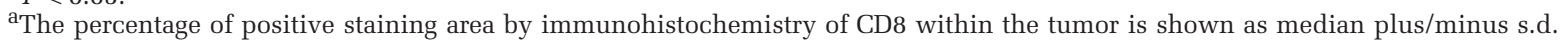

PD-L1 expression in cancer cells or stromal cells showed no correlation with survival in EBV-negative GC (data not shown).

\section{FISH Analysis of the PD-L1 Gene in EBVaGC}

FISH analysis of the $P D-L 1$ gene was applied to the sections of the TMAs that contained each representative lesion from 44 EBVaGC cases (Table 5). Gene amplification of $P D-L 1$ was observed in all five lesions containing PD-L1-positive cells with an I-score of $2(11.4 \%)$, and several dozen red signals, representing CD274 (PD-L1), were observed compared with green signals, representing CEN9q, in the nuclei of cancer cells (the median CD274/CEN9q ratio was 11.25 , range $8.45-14.55)$. The cells with multiple $P D-L 1$ signals appeared to be scattered in three cases, whereas they formed solid nests in two cases. By contrast, in the unamplified samples, 

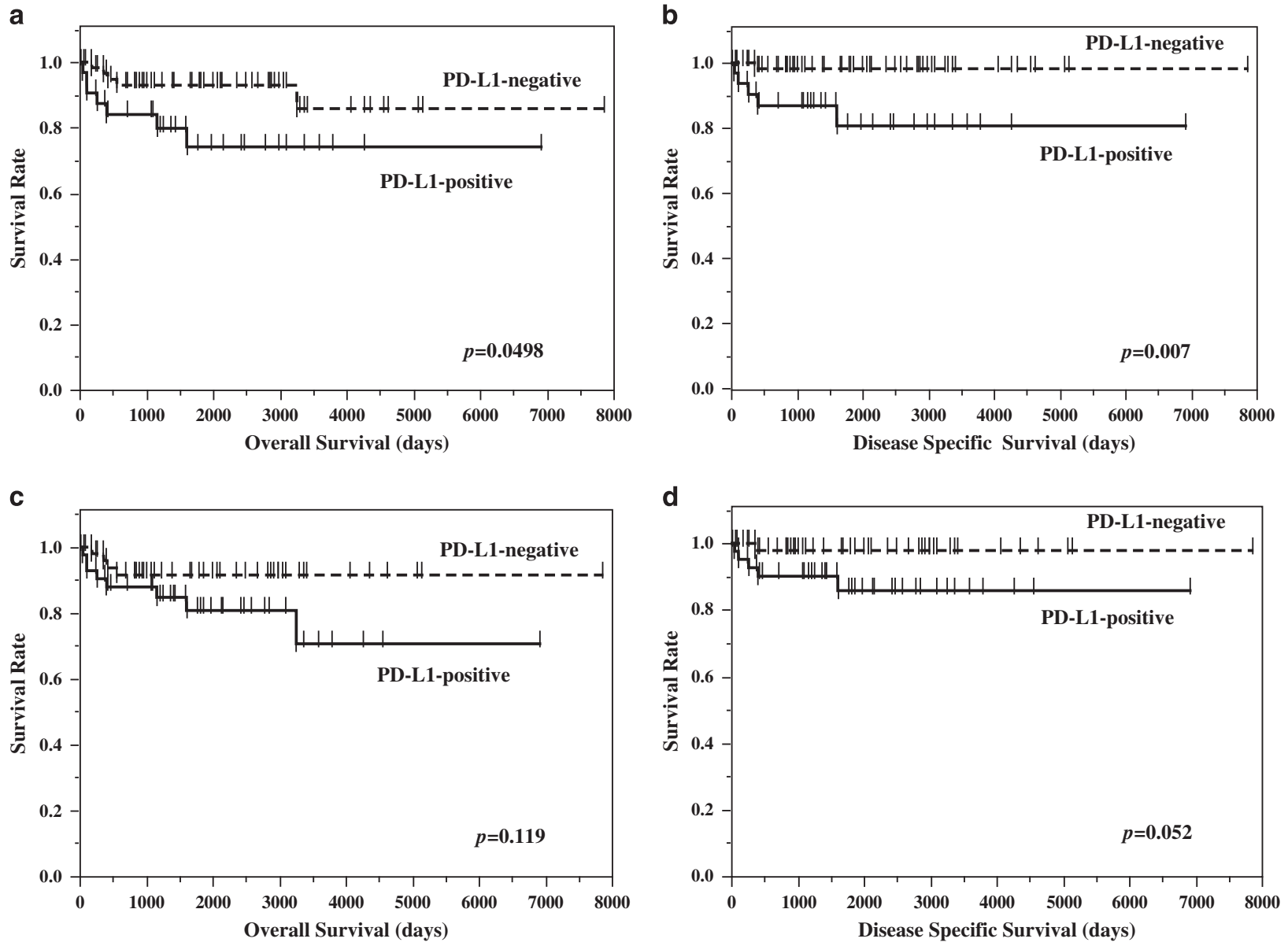

Figure 4 Survival analysis based on the expression of programmed death-ligand 1 (PD-L1) in cancer cells and their microenvironment in Epstein-Barr virus-associated gastric cancer (EBVaGC). Kaplan-Meier survival curves of EBVaGC, analyzing the effect of PD-L1 expression in cancer cells (a and b) and in stromal immune cells (c and d). (a and c) Overall survival and (b and $\mathbf{d}$ ) disease-specific survival. Expression of PD-L1 in cancer cells correlates with a poorer prognostic outcome for both overall and disease-specific survival. The infiltration of PD-L1+ immune cells also shows a tendency towards poor prognosis, but this was not statistically significant.

Table 4 Multivariate analysis of overall survival by the Cox proportional hazards model

\begin{tabular}{|c|c|c|c|c|c|c|}
\hline \multirow{2}{*}{ Prognostic factors } & \multicolumn{2}{|c|}{ Univariate analysis } & \multirow[b]{2}{*}{$\mathrm{P}$-value } & \multicolumn{2}{|c|}{ Multivariate analysis } & \multirow[b]{2}{*}{$P$-value } \\
\hline & Hazard ratio & $\begin{array}{l}95 \% \text { Confidence } \\
\text { interval }\end{array}$ & & Hazard ratio & $\begin{array}{l}95 \% \text { Confidence } \\
\text { interval }\end{array}$ & \\
\hline PD-L1 (positive) & 2.99 & $0.95-10.1$ & 0.060 & 2.69 & $0.85-9.12$ & 0.090 \\
\hline Histological type (diffuse) & 2.69 & $0.80-12.1$ & 0.113 & - & - & - \\
\hline $\begin{array}{l}\text { Tumor invasion depth (pT1b+pT2 } \\
+ \text { pT3+pT4) }\end{array}$ & 4.00 & $1.19-18.0$ & $0.024^{*}$ & - & - & - \\
\hline Venous invasion (present) & 4.13 & $1.23-18.6$ & $0.021^{*}$ & - & - & - \\
\hline Lymphatic invasion (present) & 3.47 & $1.11-11.8$ & $0.033^{*}$ & - & - & - \\
\hline Lymph node metastasis (present) & 4.14 & $1.32-14.0$ & $0.016^{*}$ & 3.81 & $1.21-13.0$ & $0.023^{*}$ \\
\hline
\end{tabular}

Abbreviation: PD-L1, programmed death-ligand 1.

${ }^{*} P<0.05$.

two of each of the red and green signals were observed in each tumor cell (median CD274/CEN9q ratio was 1.11, range $0.90-1.21$ ). All six PD-L1 ${ }^{+}$EBVnegative GCs showed no amplification of $P D-L 1$ (Figure 5).
To further confirm the observations in TMAs, FISH analysis was applied to the whole sections for three cases, in which immunohistochemistry revealed localized sheets of PD-L1-positive cells (I-score 2) within the tumor. On comparison of FISH and 
Table 5 Comparison of immunohistochemical staining and FISH of TMAs

PD-L1 immunohistochemistry in TMA cores

\begin{tabular}{ccccc} 
& Total & & & \\
\cline { 3 - 4 } & & I-score 0 & I-score 1 & I-score 2 \\
\hline FISH amplification & & & \\
Absent & 39 & 28 & 11 & 0 \\
Present & 5 & 0 & 0 & 5 \\
\hline
\end{tabular}

Abbreviations: FISH, fluorescent in situ hybridization; PD-L1, programmed death-ligand 1; I-score, intensity score; TMA, tissue microarray.
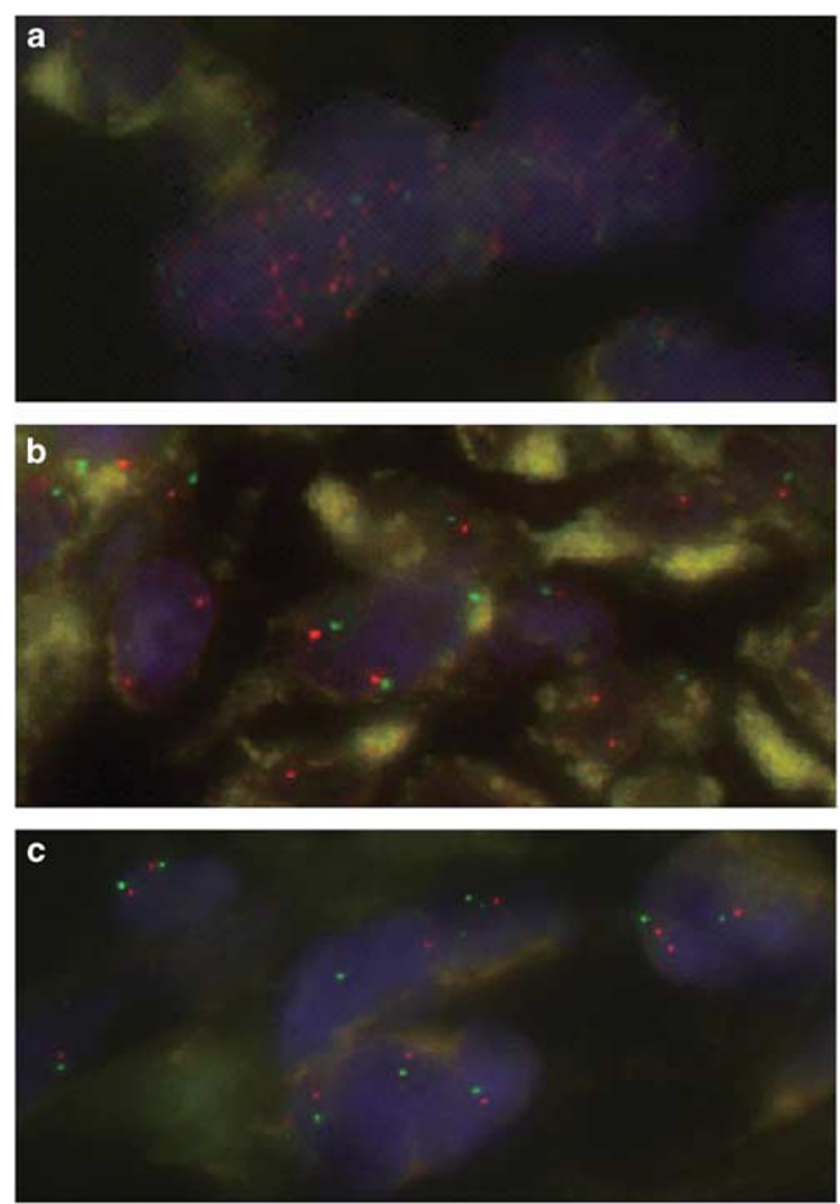

Figure 5 Fluorescent in situ hybridization (FISH) analysis of the programmed death-ligand $1(P D-L 1)$ gene. FISH analysis with a CD274 (PD-L1)/CEN9q dual-color FISH probe. Gene amplification showed several dozen PD-L1 signals (red color) and one or two CEN9q signals (green color) in each tumor cell (a). When no amplification occurred, two signals each of PD-L1 (red color) and CEN9q (green color) were observed (b and c). (a and b) EBVaGC and (c) EBV-negative gastric cancer with positive expression of PD-L1 by immunohistochemistry. These images were taken by an oil immersion lens, magnification $\times 100$.

immunohistochemistry, the cells showing $P D-L 1$ gene amplification were found to be localized in the same area as the $I$-score 2-positive cells present in sheets. No other cells showed gene amplification (Figure 6).

\section{Discussion}

PD-L1 has a critical role in evasion from antitumor immunity by attenuation of cytotoxic T-cell function through the interaction of PD-1 and PD-L1, and its expression in cancer cells has been reported to be a poor prognostic factor in a variety of cancers, including GC. ${ }^{16-21}$ It is also known that expression of PD-L1 is upregulated in various virus-related malignancies including EBV-positive diffuse large B-cell lymphoma, nasopharyngeal carcinoma, polyomavirus-positive Merkel cell carcinoma, and adult T-cell lymphoma (ATL). ${ }^{27-30}$ In the present study, immunohistochemical analysis revealed that the frequency of PD-L1 expression in cancer cells was $30 \%$ in EBVaGC, which was higher compared with that in EBV-negative GC. This predilection of PD-L1 expression in EBV-infected GC was also shown by the public database of PD-L1 mRNA expression from TCGA, and consistent with previous studies performed on relatively small numbers of cases, although the positive frequencies were variable in number. ${ }^{31-34}$ In the present study, the PD-L1-positive group of EBVaGC showed deeper invasion of the tumor and poorer prognosis, suggesting that overexpression of PD-L1 in cancer cells have a critical role in tumor progression of EBVaGC.

The microenvironment of the cancer affects cancer development and progression. In the present study, infiltration of $\mathrm{PD}-\mathrm{L}^{+}$immune cells was detected more frequently in EBVaGC than in EBV-negative GC, especially for the diffuse and intestinal subtypes. The cases harboring $\mathrm{PD}-\mathrm{L}^{+}{ }^{+}$immune cells also showed deeper invasion of the tumor in EBVaGC, although the prognostic impact of PD-L1 expression in cancer cells seemed to be superior to that of $\mathrm{PD}-\mathrm{L}^{+}{ }^{+}$immune cells. It is interesting that PD-L1 ${ }^{+}$ immune cells were also frequently detected in the MLH1-negative subtype, suggesting that this type of cancer can acquire the ability to induce PD-L1 expression despite the burden of lymphocyte infiltration. ${ }^{34,35}$ Nevertheless, both PD-1 ${ }^{+}$and PD$\mathrm{L}^{+}$immune cells were present in the stroma of PDL1-positive cancers, which suggests the possible efficacy of anti-PD-1/PD-L1 therapy in EBVaGC.

There are at least two mechanisms for the regulation of PD-L1 in tumor cells: intrinsic and adaptive immune resistance. ${ }^{36}$ The TCGA study demonstrated amplification of chromosome 9p24.1 in $15 \%$ of EBVaGC cases. $^{2}$ FISH analysis of the $P D-L 1$ gene in the present study confirmed gene amplification in $11.4 \%$ of EBVaGC cases, and this frequency was comparable to that in the TCGA study. $^{2}$ Furthermore, cancer cells with PD-L1 amplification showed an I-score of 2 for PD-L1 protein expression, indicating that gene amplification of PD-L1 directly induces overexpression in these cells. As there were no I-score 2-positive cells in EBV-negative GC and all six PD-L1 ${ }^{+}$ EBV-negative GCs were negative for amplification 

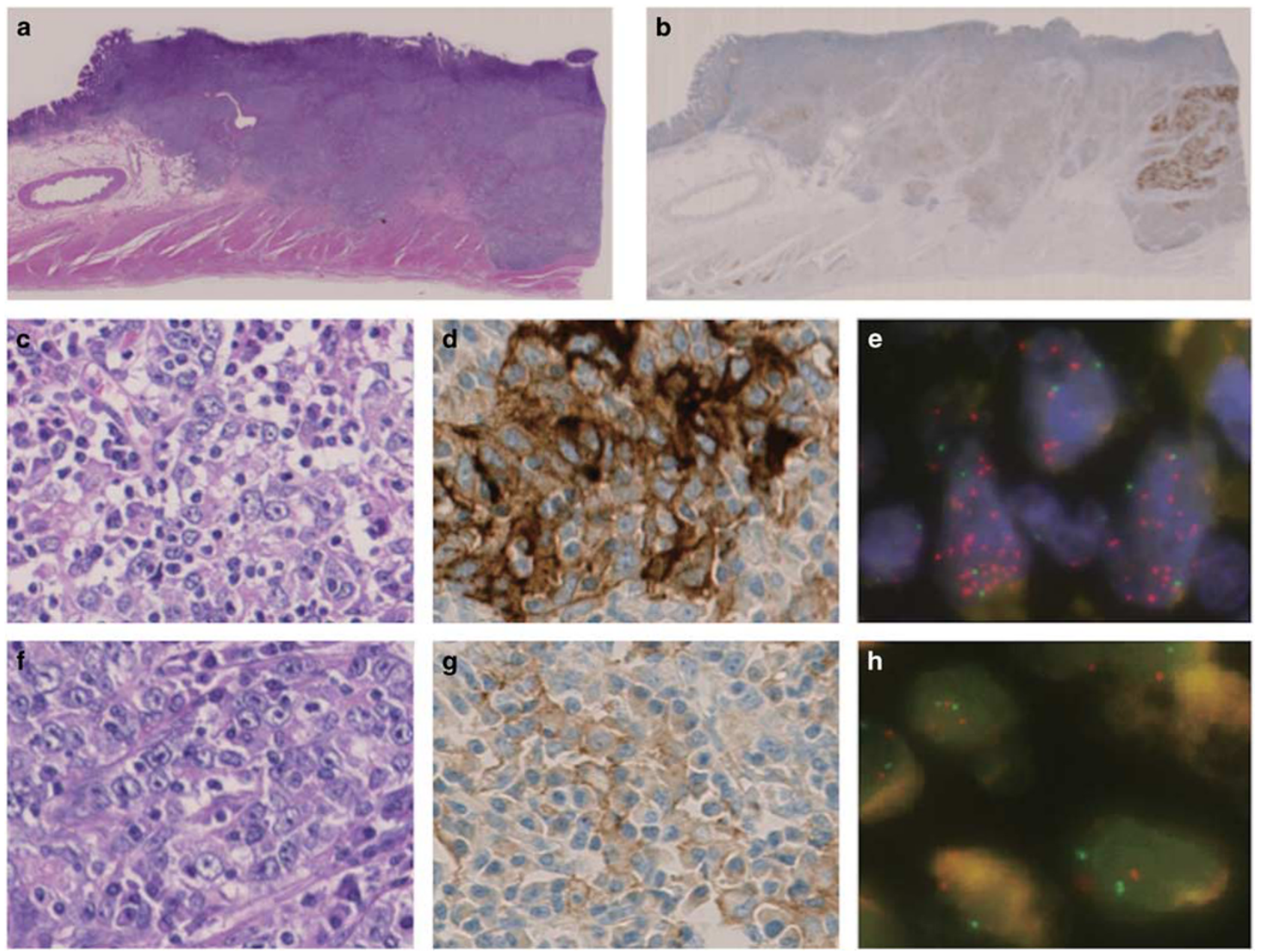

Figure 6 Distribution of cancer cells showing positive programmed death-ligand 1 (PD-L1) immunostaining and PD-L1 gene amplification. A case showing strong positive expression of PD-L1 in a limited area of the tumor (a and $\mathbf{b}$ ). This region showed an $I$-score of 2 for PD-L1 expression, compared with most other cancer cells showing an $I$-score of 1 (b). High-power view of the $I$-score 2 cells (c and d), and gene amplification results (e). High-power view of the I-score 1 cells (f and g), no amplification of the PD-L1 gene was achieved by FISH (h). (a, c, and f) Hematoxylin-eosin staining; (b, d, and g) immunohistochemistry of PD-L1; (e and h) FISH analysis of the PD-L1 gene.

of $P D-L 1$, gene amplification might occur specifically in EBV-infected cells through an unknown mechanism. It is interesting to note that genomic structural variation on deletion of the 3'-UTR of the $P D-L 1$ gene induces PD-L1 overexpression in ATL lymphoma cells infected with human T-lymphotropic virus $\mathrm{I}^{37}$ and such structural variation was observed in $2 \%$ of GCs, including EBVaGC. The $3^{\prime}$-UTR of PD-L1 mRNA is also targeted by miRNAs, some of which have been reportedly downregulated by EBV infection. ${ }^{36,38}$ The expression of PD-L1 is also increased by upregulation of the PIK3CA/Akt pathway and activation of transcriptional factors such as NF- $\mathrm{BB}$ or STAT3, all of which have been reported in EBV-infected GC cells and EBVaGC in vivo. ${ }^{8,36,39}$ Thus, viral infection itself might drive the intrinsic mechanism for overexpression of PD-L1 in this specific subtype of GC.
Adaptive immune resistance may also have a role in EBVaGC. $\mathrm{CD}^{+}$or $\mathrm{PD}-1^{+}$lymphocytes were significantly more abundant in the stroma of PD-L1-positive cases of EBVaGC in the present study and the preceding study. ${ }^{21}$ PD-L1 is reportedly upregulated on tumor cells in response to interferon- $\gamma$-secreting CD8 ${ }^{+} \mathrm{T}$ cells. ${ }^{40}$ Furthermore, it has been suggested that checkpoint inhibition is most effective in patients in which pre-existing immunity is suppressed by PD-L1. ${ }^{41}$ Therefore, combination therapy with an anti-EBV therapeutic vaccine might further augment the therapeutic effect. ${ }^{42}$ To evaluate the interactions between cancer cells and immune cells more efficiently, a double immunostaining method should be developed that preserves high staining sensitivity.

A recent phase $1 \mathrm{~b}$ clinical trial of the anti-PD-1 antibody pembrolizumab in GC revealed promising antitumor activity. ${ }^{43}$ It is therefore important to 
determine screening methods to select the patients who will benefit from immunotherapy, which poses the question of whether PD-L1 protein overexpression in cancer cells or their microenvironment in EBVaGC is more suitable for predicting the therapeutic effect. As for EBV-negative GC, the rarity of PD-L1 expression in cancer cells suggests that PD-L1 expression in stromal cells may be a more effective candidate for predicting the efficacy of anti-PD-1/PD-L1 therapy. In fact, a study of pembrolizumab in GC patients revealed that cancer cells did not express PD-L1 in seven of eight responders, whereas immune cells at least focally expressed PD-L1 in all eight responders. ${ }^{43} \mathrm{~A}$ recent clinical trial of atezolizumab in lung cancer showed that PD-L1 expression in tumor cells or tumor-infiltrating immune cells correlated with improvement of patient survival. ${ }^{44}$ These results suggest the importance of PD-L1 expression in immune cells as well as tumor cells. A potential problem with this strategy is selecting suitable antibodies for screening. ${ }^{18-21}$ We used a rabbit monoclonal anti-PD-L1 antibody (clone E1L3N) in the present study, and found that the staining intensity with stromal immune cells was relatively weak. Several studies have reported variable expression patterns of PD-L1 depending on the antibodies used for immunohistochemistry, and it has yet to be determined which antibody is appropriate for the assessment of PD-L1 expression when selecting patients for PD-L1-targeting therapy. ${ }^{45,46}$ We preliminarily compared different antibody clones (E1L3N, SP263, SP142, and 28-8) in representative cases. We found that the proportion of PD-L1 ${ }^{+}$cancer cells using SP263 antibody was almost the same as the E1L3N antibody, and larger than the proportions detected by the SP142 or $28-8$ antibody, although the SP263 antibody showed the strongest intensity (data not shown). Another problem is the heterogeneity of the tumor and its microenvironment. ${ }^{47}$ Cautious standardization will be necessary to determine the grade of $\mathrm{PD}-\mathrm{L}^{+}$ immune cells in the cancer stroma, especially when only biopsy specimens are available. For example, the false-negative detection rate of immunohistochemistry of HER2 in GC was estimated at around $0.21 \%$ with four biopsies and approached $0 \%$ with five biopsies. ${ }^{48}$ To predict the effect of anti-PD-1/PDL1 therapy in GC, further studies are mandatory to determine appropriate number of biopsies, selection of antibody, and evaluation method of immunohistochemistry.

In conclusion, PD-L1 expression in tumor cells is a frequent characteristic of EBVaGC and correlates with poor prognosis. Gene amplification of $P D-L 1$ is an important mechanism of PD-L1 expression in EBVaGC, and can occur during clonal evolution at a late stage of cancer progression. Although the results in the present study should be validated with larger age-stage matched cohort in future studies, our findings indicate that PD-L1 should be a therapeutic target of EBVaGC. Further studies are necessary to reveal the mechanism of PD-L1 overexpression, which contributes to the immunotherapy of EBVaGC, a specific subtype of GC infected with a human oncogenic virus.

\section{Acknowledgments}

We thank Kei Sakuma, Harumi Yamamura, Kimiko Takeshita, and Aiko Nishimoto for excellent technical assistance. This study was supported by a grantin-aid for scientific research (KAKENHI) (grant numbers 26253021 (to MF), 26860232 (to HA), and 16K19074 (to HA)) from the Japan Society for the Promotion of Science.

\section{Disclosure/conflict of interest}

The authors declare no conflict of interest.

\section{References}

1 Bosman FT, Carneiro F, Hruban RH et al. WHO Classification of Tumours of the Digestive System, 4th edn. IARC Press: Lyon, France, 2010.

2 The Cancer Genome Atlas Research Network. Comprehensive molecular characterization of gastric adenocarcinoma. Nature 2014;513:202-209.

3 Wang K, Yuen ST, Xu J, et al. Whole-genome sequencing and comprehensive molecular profiling identify new driver mutations in gastric cancer. Nat Genet 2014;46:573-582.

4 Shinozaki-Ushiku A, Kunita A, Fukayama M. Update on Epstein-Barr virus and gastric cancer [review]. Int J Oncol 2015;46:1421-1434.

5 Abe H, Kaneda A, Fukayama M. Epstein-Barr virusassociated gastric carcinoma: use of host cell machineries and somatic gene mutations. Pathobiology 2015;82:212-223.

6 Fukayama M, Hino R, Uozaki H. Epstein-Barr virus and gastric carcinoma: virus-host interactions leading to carcinoma. Cancer Sci 2008;99:1726-1733.

7 Song HJ, Srivastava A, Lee J, et al. Host inflammatory response predicts survival of patients with EpsteinBarr virus-associated gastric carcinoma. Gastroenterology 2010;139:84-92.

8 Hino R, Uozaki H, Murakami N, et al. Activation of DNA methyltransferase 1 by EBV latent membrane protein 2A leads to promoter hypermethylation of PTEN gene in gastric carcinoma. Cancer Res 2009;69: 2766-2774.

9 Matsusaka K, Kaneda A, Nagae G, et al. Classification of Epstein-Barr virus-positive gastric cancers by definition of DNA methylation epigenotypes. Cancer Res 2011;71:7187-7197.

10 Iwai $\mathrm{Y}$, Ishida $\mathrm{M}$, Tanaka $\mathrm{Y}$, et al. Involvement of PD- L1 on tumor cells in the escape from host immune system and tumor immuno- therapy by PD-L1 blockade. Proc Natl Acad Sci USA 2002;99: 12293-12297.

11 Blank C, Gajewski TF, Mackensen A. Interaction of PD-L1 on tumor cells with PD-1 on tumor-specific $\mathrm{T}$ cells as a mechanism of immune evasion: 
implications for tumor immunotherapy. Cancer Immunol Immunother 2005;54:307-314.

12 Francisco LM, Sage PT, Sharpe AH. The PD-1 pathway in tolerance and autoimmunity. Immunol Rev 2010;236:219-242.

13 Ohaegbulam KC, Assal A, Lazar-Molnar E, et al. Human cancer immunotherapy with antibodies to the PD-1 and PD-L1 pathway. Trends Mol Med 2015;21: 24-33.

14 Topalian SL, Hodi FS, Brahmer JR, et al. Safety, activity, and immune correlates of anti-PD-1 antibody in cancer. N Engl J Med 2012;366:2443-2454.

15 Szno M, Chen L. Antagonist antibodies to PD-1 and B7H1 (PD-L1) in the treatment of advanced human cancer. Clin Cancer Res 2013;19:1021-1034.

16 Hino R, Kabashima K, Kato Y, et al. Tumor cell expression of programmed cell death-1 ligand 1 is a prognostic factor for malignant melanoma. Cancer 2010;116:1757-1766.

17 Velcheti V, Schalper KA, Carvajal DE, et al. Programmed death ligand-1 expression in non-small cell lung cancer. Lab Invest 2014;94:107-116.

18 Zhang Lin, Miaozhen Qiu, Ying Jin, et al. Programmed cell death ligand 1 (PD-L1) expression on gastric cancer and its relationship with clinicopathologic factors. Int J Clin Exp Pathol 2015;8:11084-11091.

19 Qing Y, Li Q, Ren T, et al. Upregulation of PD-L1 and APE1 is associated with tumorigenesis and poor prognosis of gastric cancer. Drug Des Devel Ther 2015;9:901-909.

20 Kim JW, Nam KH, Ahn SH, et al. Prognostic implications of immunosuppressive protein expression in tumors as well as immune cell infiltration within the tumor microenvironment in gastric cancer. Gastric Cancer 2016;19:42-52.

21 Thompson ED, Zahurak M, Murphy A, et al. Patterns of PD-L1 expression and CD8 T cell infiltration in gastric adenocarcinomas and associated immune stroma. Gut; available from: URL: http://gut.bmj.com/content/early/ 2016/01/22/gutjnl-2015-310839.long.

22 Liu YX, Wang XS, Wang YF, et al. Prognostic significance of PD-L1 expression in patients with gastric cancer in East Asia: a meta-analysis. Onco Targets Ther 2016;9:2649-2654.

23 Cerami E, Gao J, Dogrusoz U, et al. The cBio cancer genomics portal: an open platform for exploring multidimensional cancer genomics data. Cancer Discov 2012;2:401-404.

24 Gao J, Aksoy BA, Dogrusoz U, et al. Integrative analysis of complex cancer genomics and clinical profiles using the cBioPortal. Sci Signal; available from: URL: http://stke.sciencemag.org/content/sigtrans/6/269/pl1. full.pdf.

25 Lauren P. The two histological main types of gastric carcinoma: diffuse and so-called intestinal-type carcinoma. An attempt at a histo-clinical classification. Acta Pathol Microbiol Scand 1965;64:31-49.

26 Abe H, Morikawa T, Saito R, et al. CD66b-positive tumor-associated neutrophils in Epstein-Barr virus associated gastric carcinoma. A comparative study with CD8-positive cytotoxic T-lymphocytes. Virchows Arch 2016;468:539-548.

27 Chen BJ, Chapuy B, Ouyang J, et al. PD-L1 expression is characteristic of a subset of aggressive B-Cell lymphomas and virus-associated malignancies. Clin Cancer Res 2013;19:3462-3473.
28 Fang W, Zhang J, Hong S, et al. EBV-driven LMP1 and IFN- $\gamma$ up-regulate PD-L1 in nasopharyngeal carcinoma: Implications for oncotargeted therapy. Oncotarget 2014:5:12189-12202.

29 Lipson EJ, Vincent JG, Loyo M, et al. PD-L1 expression in the Merkel cell carcinoma microenvironment: association with inflammation, Merkel cell polyomavirus and overall survival. Cancer Immunol Res 2013;1: 54-63.

30 Kozako T, Yoshimitsu M, Fujiwara H, et al. PD-1/PD-L1 expression in human T-cell leukemia virus type 1 carriers and adult T-cell leukemia/lymphoma patients. Leukemia 2009;23:375-382.

31 Derks S, Liao X, Chiaravalli AM, et al. Abundant PD-L1 expression in Epstein-Barr virus-infected gastric cancers. Oncotarget 2016;7:32925-32932.

32 Boger C, Behrens HM, Mathiak M, et al. PD-L1 is an independent prognostic predictor in gastric cancer of Western patients. Oncotarget 2016;7: 24269-24283.

$33 \mathrm{Li} \mathrm{Z}$, Lai Y, Sun L, et al. PD-L1 expression is associated with massive lymphocyte infiltration and histology in gastric cancer. Hum Pathol 2016;55: 182-189.

34 Inaguma S, Wang Z, Lasota J, et al. Comprehensive immunohistochemical study of programmed cell death ligand 1 (PD-L1): analysis in 5536 cases revealed consistent expression in trophoblastic tumors. Am J Surg Pathol 2016;40:1133-1142.

35 Grogg KL, Lohse CM, Pankratz VS, et al. Lymphocyterich gastric cancer: associations with Epstein-Barr virus, microsatellite instability, histology, and survival. Mod Pathol 2003;16:641-651.

36 Chen J, Jiang CC, Jin L, et al. Regulation of pd-l1: a novel role of pro-survival signalling in cancer. Ann Oncol 2016;27:409-416.

37 Kataoka K, Shiraishi Y, Takeda Y, et al. Aberrant PD-L1 expression through 3'-UTR disruption in multiple cancers. Nature 2016;534:402-406.

38 Shinozaki A, Sakatani T, Ushiku T, et al. Downregulation of microRNA-200 in EBV-associated gastric carcinoma. Cancer Res 2010;70:4719-4727.

39 Hino R, Uozaki H, Inoue Y, et al. Survival advantage of EBV-associated gastric carcinoma: survivin upregulation by viral latent membrane protein $2 \mathrm{~A}$. Cancer Res 2008;68:1427-1435.

40 Spranger S, Spaapen RM, Zha Y, et al. Up-regulation of PD-L1, IDO, and T(regs) in the melanoma tumor microenvironment is driven by CD8(+) T cells. Sci Transl Med 2013;5:200ra116.

41 Tumeh PC, Harview CL, Yearley JH, et al. PD-1 blockade induces responses by inhibiting adaptive immune resistance. Nature 2014;515:568-571.

42 Taylor GS, Steven NM. Therapeutic vaccination strategies to treat nasopharyngeal carcinoma. Chin Clin Oncol 2016;5:23.

43 Muro K, Chung HC, Shankaran V, et al. Pembrolizumab for patients with PD-L1-positive advanced gastric cancer (KEYNOTE-012): a multicentre, open-label, phase 1b trial. Lancet Oncol 2016;17:717-726.

44 Fehrenbacher L, Spira A, Ballinger $\mathrm{M}$, et al. Atezolizumab versus docetaxel for patients with previously treated non-small-cell lung cancer (POPLAR): a multicentre, open-label, phase 2 randomised controlled trial. Lancet 2016;387: 1837-1846. 
45 Smith J, Robida MD, Acosta K, et al. Quantitative and qualitative characterization of two PD-L1 clones: SP263 and E1L3N. Diagn Pathol 2016; 11:44.

46 Kerr KM, Tsao MS, Nicholson AG, et al. Programmed death-ligand 1 immunohistochemistry in lung cancer: in what state is this art? J Thorac Oncol 2015;10: 985-989.
47 McLaughlin J, Han G, Schalper KA, et al. Quantitative assessment of the heterogeneity of PD-L1 expression in non-small-cell lung cancer. JAMA Oncol 2016;2:46-54.

48 Ye P, Zhang M, Fan S, et al. Intra-tumoral heterogeneity of HER2, FGFR2, cMET and ATM in gastric cancer: Optimizing personalized healthcare through innovative pathological and statistical analysis. PLoS One 2015;10:e0143207.

Supplementary Information accompanies the paper on Modern Pathology website (http://www.nature.com/ modpathol) 